\title{
MINKOWSKI'S FUNDAMENTAL INEQUALITY FOR REDUCED POSITIVE QUADRATIC FORMS (II)
}

\author{
E. S. BARNES
}

(Received 10 May 1978)

\begin{abstract}
A convex polytope $\mathscr{D}(\alpha)$ was defined in Barnes (1978) as the set of Minkowski-reduced forms with prescribed diagonal coefficients $\alpha_{1}, \alpha_{2}, \ldots, \alpha_{n}$. A local minimum of the determinant $D(f)$ over $\mathscr{D}(\alpha)$ must occur at a vertex of $\mathscr{D}(\alpha)$. Here a criterion is obtained for a given vertex to provide a local minimum, completely analogous to Voronoi's criterion for a perfect form to be extreme.
\end{abstract}

Subject classification (Amer. Math. Soc. (MOS) 1970): primary 10 E 25; secondary 10 E 20.

\section{Introduction}

We use the definitions introduced in Part I (Barnes, 1978): $\mathscr{M}$ is the polyhedral cone of Minkowski-reduced forms in $n$ variables; $\mathscr{D}(\alpha)$ is the subset of $\mathscr{M}$ consisting of those positive forms $f(\mathbf{x})=\sum_{1}^{n} a_{i j} x_{i} x_{j}$ for which

$$
a_{i l}=\alpha_{i} \quad(i=1, \ldots, n) \text {, }
$$

where necessarily

$$
0<\alpha_{1} \leqslant \alpha_{2} \leqslant \ldots \leqslant \alpha_{n}
$$

$\mathscr{D}(\alpha)$ is in fact a convex polytope.

The convexity of the determinantal surface $D(f)=$ constant implies that the minimum value of $D$ over $\mathscr{D}(\alpha)$, or indeed any local minimum, is attained only at a vertex of $\mathscr{D}(\alpha)$. However, it is not necessarily true that a vertex $v$ of $\mathscr{D}(\alpha)$ provides a local minimum of $D(f)$ for $f \in \mathscr{D}(\alpha)$; a vertex for which this is true we call extreme with respect to $\mathscr{D}(\alpha)$, or, for brevity, $\mathscr{D}$-extreme.

The main purpose of this article is to establish an analogue of Voronoï's (1907) well-known criterion for a form to be extreme in the classical sense, namely that it be perfect and eutactic. The analogue of a perfect form is clearly a vertex of $\mathscr{D}(\alpha)$.

(C) Copyright Australian Mathematical Society 1979

Copyright. Apart from any fair dealing for scholarly purposes as permitted under the Copyright Act, no part of this JOURNAL may be reproduced by any process without written permission from the Treasurer of the Australian Mathematical Society. 
We construct the analogue of a eutactic form as follows. Recall first that $f$ is Minkowski-reduced if and only if, for all $i=1, \ldots, n$ and all integral $\mathbf{x}$,

$$
f(x) \geqslant a_{i i} \text { if g.c.d. }\left(x_{i}, x_{i+1}, \ldots, x_{n}\right)=1 .
$$

If $f \in \mathscr{D}(\alpha)$, denote by $\mathbf{m}_{k}(k=1, \ldots, t)$ those $\mathbf{x}$, other than unit vectors, for which equality holds in (1.3) (as usual, we identify $\mathbf{x}$ and $-\mathbf{x}$ in such statements). Then we say that $f$ is $\mathscr{D}$-eutactic if its adjoint $F$ is expressible in the form

$$
F(\mathbf{x})=\sum_{1}^{n} A_{i j} x_{i} x_{j}=\sum_{1}^{t} \rho_{k}\left(\mathbf{m}_{k}^{\prime} \mathbf{x}\right)^{2}+\sum_{1}^{n} \sigma_{i} x_{i}^{2},
$$

where the $\rho_{k}, \sigma_{i}$ are real numbers and

$$
\rho_{k}>0 \quad(k=1, \ldots, t) .
$$

THEOREM 1. A form $f \in \mathscr{D}(\alpha)$ is $\mathscr{D}$-extreme if and only if it is a vertex of $\mathscr{D}(\alpha)$ and is $\mathscr{D}$-eutactic.

The proof of this theorem will be based on the ideas used in the proof of Voronoi's Theorem given in Barnes (1957); as there, we need

TheOREM 2 (Stiemke, 1915). The system of linear inequalities

$$
\sum_{j=1}^{p} t_{i j} u_{j}=0, \quad u_{j}>0 \quad(i=1, \ldots, m ; j=1, \ldots, p)
$$

has a solution $\mathbf{u}$ if and only if every solution $\mathbf{v}$ of the dual system

$$
\sum_{i=1}^{m} t_{i j} v_{i} \geqslant 0 \quad(j=1, \ldots, p)
$$

satisfies

$$
\sum_{i=1}^{m} t_{i j} v_{i}=0 \quad(j=1, \ldots, p) .
$$

We shall also need the following simple geometrical result:

Lemma 1. Let $K$ be an $N$-dimensional convex set and $\mathbf{p}$ a point of $b d K$, in a neighbourhood of which $K$ is strictly convex and $b d K$ is smooth. Let $h$ be the tangent plane to $K$ at $\mathbf{p}$ and $h^{+}$the open half-space determined by $h$ and containing int $K$. Let $P$ be a convex polytope with a vertex at $\mathbf{p}$, and suppose that the whole of $P$ in some punctured neighbourhood of $\mathbf{p}$ lies in $h^{+}$. Then there exists a punctured neighbourhood $B$ of $\mathbf{p}$ such that if $\mathbf{q} \in B \cap P$ then $\mathbf{q} \in$ int $K$.

\section{Proof of Theorem 1}

We begin with the appropriate analogue of the Lemma of Barnes (1957):

Lemma 2. If $f \in \mathscr{D}(\alpha)$, then $f$ is $\mathscr{D}$-extreme if and only if there exists no non-trivial quadratic form $g(\mathbf{x})=\sum_{1}^{n} b_{i j} x_{i} x_{j}$ satisfying 


$$
\begin{aligned}
& g\left(\mathbf{e}_{i}\right)=0 \quad(i=1, \ldots, n), \\
& g\left(\mathbf{m}_{k}\right) \geqslant 0 \quad(k=1, \ldots, t), \\
& (F, g)=\sum A_{i j} b_{i j} \leqslant 0 .
\end{aligned}
$$

Proof. (i) Suppose that (2.1), (2.2), (2.3) have a non-trivial solution $g$. Choose one with, say $\max \left|b_{i j}\right|=1$, and set $f^{\prime}=f+\varepsilon g$ where $\varepsilon>0$ is small.

Then $f^{\prime} \in \mathscr{D}(\alpha)$. For firstly

$$
f^{\prime}\left(\mathbf{e}_{i}\right)=f\left(\mathbf{e}_{i}\right)=\alpha_{i} \quad(i=1, \ldots, n) ;
$$

and, for any $k=1, \ldots, t$ for which $\mathbf{m}_{k}$ satisfies (1.3) with equality for some $i$,

$$
f^{\prime}\left(\mathbf{m}_{k}\right)=f\left(\mathbf{m}_{k}\right)+\varepsilon g\left(\mathbf{m}_{k}\right)=\alpha_{i}+\varepsilon g\left(\mathbf{m}_{k}\right) \geqslant \alpha_{i} .
$$

Next, there exist only finitely many other $\mathbf{x}$ in (1.3) which are necessary to specify $\mathscr{M}$ and, for all of these, $f(\mathbf{x})>a_{i i}=\alpha_{i}$, whence also $f^{\prime}(\mathbf{x})>\alpha_{i}$ if $\varepsilon$ is sufficiently small.

Now the tangent plane $h$ to the determinantal surface (using $\varphi$ as current coordinates in the coefficient space) at $f$ is

$$
(F, \varphi)=(F, f)=n \operatorname{det} f .
$$

Since

$$
\left(F, f^{\prime}\right)=(F, f)+\varepsilon(F, g) \leqslant(F, f),
$$

it follows that $f^{\prime}$ lies in the closed half-space opposite to that containing the surface $\operatorname{det} \varphi=\operatorname{det} f$; hence, since this surface is strictly convex and $f^{\prime} \neq f, \operatorname{det} f^{\prime}<\operatorname{det} f$.

It follows from these results that $f$ is not $\mathscr{D}$-extreme.

(ii) Suppose that (2.1), (2.2), (2.3) have only the trivial solution; let $f^{\prime}=f+g$ $(g \not \equiv)$ be any form in $\mathscr{D}(\alpha)$ close to $f$. Then

$$
\begin{aligned}
& f^{\prime}\left(\mathbf{e}_{i}\right)=\alpha_{i}=f\left(\mathbf{e}_{i}\right), \quad \text { so that } g\left(\mathbf{e}_{i}\right)=0 \quad(i=1, \ldots, n) \text {; } \\
& f^{\prime}\left(\mathbf{m}_{k}\right) \geqslant \alpha_{i}=f\left(\mathbf{m}_{k}\right) \text {, so that } g\left(\mathbf{m}_{k}\right) \geqslant 0 \quad(k=1, \ldots, t) \text {. }
\end{aligned}
$$

Since $g$ is non-trivial, our hypothesis implies that $(2.3)$ is ialse, so that $(F, g)>0$ and

$$
\left(F, f^{\prime}\right)=(F, f)+(F, g)>(F, f) \text {. }
$$

We now apply Lemma 1 , taking $K$ to be the determinantal body $\operatorname{det} \varphi \geqslant \operatorname{det} f, h$ the tangent plane $(F, \varphi)=(F, f)$, and $P$ the polytope $\mathscr{D}(\alpha)$. Using in particular (2.4), we see that the hypotheses of the lemma hold and it follows that, if $f^{\prime}$ is sufficiently close to $f$ in $\mathscr{D}(\alpha)$, but distinct from $f$, then $\operatorname{det} f^{\prime}>\operatorname{det} f$. Thus $f$ is $\mathscr{D}$-extreme. 
Proof of TheOrem 1. Using the coefficients $b_{i j}$ of the form $g$, write (2.1)-(2.3) as

$$
\left\{\begin{aligned}
& b_{i l} \geqslant 0 \\
&-b_{i l} \geqslant 0(i=1, \ldots, n), \\
& \sum_{1}^{n} b_{i j} m_{i k} m_{j k} \geqslant 0 \quad(k=1, \ldots, t), \\
&-\sum A_{i j} b_{i j} \geqslant 0 .
\end{aligned}\right.
$$

We identify this with the system (1.7), with $b_{11}, b_{12}, \ldots, b_{n n}$ playing the part of the variables $v_{1}, v_{2}, \ldots, v_{m}$. Then (1.6) becomes, using variables $\lambda_{i}, \mu_{i}(i=1, \ldots, n)$, $\rho_{k}(k=1, \ldots, t), v$,

$$
\left\{\begin{array}{c}
\lambda_{i} \delta_{i j}-\mu_{i} \delta_{i j}+\sum_{1}^{t} \rho_{k} m_{i k} m_{j k}-v A_{i j}=0 \quad(i, j=1, \ldots, n), \\
\lambda_{i}>0, \quad \mu_{i}>0, \quad \rho_{k}>0, \quad v>0 .
\end{array}\right.
$$

(a) Suppose that $f$ is $\mathscr{D}$-extreme. By Lemma 2, every solution of (2.5) is trivial and so certainly has equality throughout; it follows at once that $f$ is a vertex of $\mathscr{D}(\alpha)$. Also, by Stiemke's Theorem, (2.6) has a solution; dividing through by $v$, we may suppose that the solution has $v=1$; multiplying by $x_{l} x_{j}$ and summing, we obtain

$$
F(\mathbf{x})=\sum_{1}^{n} A_{i j} x_{i} x_{j}=\sum_{1}^{t} \rho_{k}\left(\mathbf{m}_{k}^{\prime} \mathbf{x}\right)^{2}+\sum_{1}^{n}\left(\lambda_{i}-\mu_{i}\right) x_{i}^{2}
$$

which gives (1.4), with (1.5), noting that $\sigma_{i}=\lambda_{t}-\mu_{i}$ is unrestricted in sign.

(b) Suppose next that $f$ is a vertex of $\mathscr{D}(\alpha)$ and is $\mathscr{D}$-eutactic. Then (2.6) has a solution and so, by Stiemke's Theorem, any solution $g$ of (2.5) satisfies (2.5) with equality throughout; since $f$ is a vertex of $\mathscr{D}(\alpha)$ it then follows that $g \equiv 0$. It now follows from Lemma 2 that $f$ is $\mathscr{D}$-extreme.

\section{An example}

As noted in Part $I$, the quaternary form

$$
f(\mathbf{x})=a x_{1}^{2}+a x_{1} x_{2}-a x_{1} x_{3}-a x_{1} x_{4}+b x_{2}^{2}-b x_{2} x_{4}+c x_{3}^{2}+c x_{3} x_{4}+d x_{4}^{2}
$$

is a vertex of $\mathscr{D}(\alpha)=\mathscr{D}(a, b, c, d)$ which is however not $\mathscr{D}$-extreme for some values of $a, b, c, d$ (where we still of course assume (1.2), that is

$$
0<a \leqslant b \leqslant c \leqslant d) \text {. }
$$

THEOREM 2. Suppose that $a<b$. Then the form (3.1), subject to (3.2), is $\mathscr{D}$-extreme if and only if

$$
a d<b c \text {. }
$$


Proof. It is easily verified that for the form (3.1), neglecting unit vectors, there are just seven relations (1.3) that hold with equality, namely

$$
\begin{gathered}
f(-1,1,0,0)=b, \quad f(1,0,1,0)=c, \\
f(1,0,0,1)=f(0,1,0,1)=f(0,0,-1,1)=f(0,1,-1,1)=f(-1,1,-1,1)=d .
\end{gathered}
$$

These suffice to establish that $f$ is a vertex of $\mathscr{D}(\alpha)$.

[Note: If $a=b$, there is one further relation $f(1,-1,1,0)=c$ and the following analysis therefore does not apply.]

The identity (1.4) is now

$$
\begin{aligned}
\sum_{1}^{4} A_{i j} x_{i} x_{j}= & \rho_{1}\left(-x_{1}+x_{2}\right)^{2}+\rho_{2}\left(x_{1}+x_{3}\right)^{2}+\rho_{3}\left(x_{1}+x_{4}\right)^{2} \\
& +\rho_{4}\left(x_{2}+x_{4}\right)^{2}+\rho_{5}\left(-x_{3}+x_{4}\right)^{2}+\rho_{6}\left(x_{2}-x_{3}+x_{4}\right)^{2} \\
& +\rho_{7}\left(-x_{1}+x_{2}-x_{3}+x_{4}\right)^{2}+\sum_{1}^{4} \sigma_{i} x_{i}^{2},
\end{aligned}
$$

and this yields

$$
\begin{aligned}
\rho_{4} & =A_{23}+A_{24}=\frac{1}{8} a(a b+a c-2 a d-2 b c)+\frac{1}{8} a(-a b-a c+4 b c) \\
& =\frac{1}{4} a(b c-a d) .
\end{aligned}
$$

If now $a d \geqslant b c$, then $\rho_{4} \leqslant 0$ and so, by Theorem $2, f$ is not $\mathscr{D}$-extreme. If however $a d<b c$, it is easy to verify that (3.4) is soluble with all $\rho_{k}>0$; one may take $\rho_{7}$ sufficiently small and positive and then determine $\rho_{1}, \ldots, \rho_{6}$ from the relations

$$
\begin{aligned}
& \rho_{1}=-A_{12}-\rho_{7}, \quad \rho_{2}=A_{13}-\rho_{7}, \quad \rho_{3}=A_{14}+\rho_{7}, \\
& \rho_{4}=\rho_{5}=\frac{1}{4} a(b c-a d), \quad \rho_{6}=-A_{23}-\rho_{7} .
\end{aligned}
$$

\section{A refinement of Theorem 1}

Among the inequalities (1.3) there is a finite set which implies all remaining inequalities; that is, there is a finite set $S$ of vectors $\mathbf{x}$ for which the corresponding equations $f(\mathbf{x})=a_{i i}$ define the facets of $\mathscr{M}$. In determining $\mathscr{D}(\boldsymbol{\alpha})$ and its vertices, it suffices of course to consider only this minimal set of inequalities. However, the application of Theorem 1 is complicated by the need to know all the examples of equality in (1.3) for a given form $f$. Fortunately it turns out to be necessary, in testing whether a vertex $f$ of $\mathscr{D}(\alpha)$ is $\mathscr{D}$-extreme, to consider only vectors in $S$ :

THEOREM 3. Let $f$ be a vertex of $\mathscr{D}(\boldsymbol{\alpha})$ and $\mathbf{m}_{k}(k=1, \ldots, r)(r \leqslant t)$ be the vectors of $S$, other than unit vectors, for which equality holds in (1.3) Then $f$ is $\mathscr{D}$-extreme if and only if $f$ is eutactic with respect to this set of vectors, that is, if

$$
F(\mathbf{x})=\sum_{1}^{n} A_{i j} x_{i} x_{j}=\sum_{i}^{r} \rho_{k}^{\prime}\left(\mathbf{m}_{k}^{\prime} \mathbf{x}\right)^{2}+\sum_{1}^{n} \sigma_{i}^{\prime} x_{i}^{2}
$$

for some real $\rho_{k}^{\prime}, \sigma_{i}^{\prime}$ satisfying $\rho_{k}^{\prime}>0(k=1, \ldots, r)$. 
For the proof of Theorem 4 it clearly suffices to establish the corresponding strengthening of Lemma 2, namely

LEMMA 3. Let $f \in \mathscr{D}(\alpha)$ and let $\mathbf{m}_{k}(k=1, \ldots, r)$ be those $\mathbf{x} \in S$, other than unit vectors, for which equality holds in (1.3). Then $f$ is $\mathscr{D}$-extreme if and only if there exists no non-trivial quadratic form $g$ satisfying

$$
g\left(\mathbf{e}_{i}\right)=0 \quad(i=1, \ldots, n), \quad g\left(\mathbf{m}_{k}\right) \geqslant 0 \quad(k=1, \ldots, r), \quad(F, g) \leqslant 0 .
$$

ProOF. Only one modification is needed in the proof given for Lemma 2, namely for the assertion in part (i) that $f^{\prime} \in \mathscr{D}(\alpha)$. We now have

$$
f^{\prime}\left(\mathbf{e}_{i}\right)=f\left(\mathbf{e}_{i}\right)=\alpha_{i} \quad(i=1, \ldots, n)
$$

and, for each $k=1, \ldots, r$ and the corresponding $i$,

$$
f^{\prime}\left(\mathbf{m}_{k}\right)=f\left(\mathbf{m}_{k}\right)+\varepsilon g\left(\mathbf{m}_{k}\right) \geqslant f\left(\mathbf{m}_{k}\right)=\alpha_{i} .
$$

Next, if $\mathbf{x} \in S$ but is not a unit vector or one of $\mathbf{m}_{1}, \ldots, \mathbf{m}_{r}$, our hypothesis implies that $f(\mathbf{x})>a_{i i}$, whence also $f^{\prime}(\mathbf{x})>a_{i i}=\alpha_{i}$ if $\varepsilon$ is sufficiently small. Since $S$ is finite, it follows that $f^{\prime}(\mathbf{x}) \geqslant \alpha_{i}$ for all $\mathbf{x} \in S$ (and corresponding $i$ ), and hence, by definition of $S$, for all integral $\mathbf{x}$.

\section{References}

E. S. Barnes (1978), 'On Minkowski's fundamental inequality for reduced positive quadratic forms (I)', J. Austral. Math. Soc. 26, 46-52.

E. S. Barnes (1957), 'On a theorem of Voronoï', Proc. Cambridge Phil. Soc. 53, 537-539.

E. Stiemke (1915) 'Über positive Lösungen homogener linearer Gleichungen', Math. Ann. 76, 340-342.

G. Voronoï (1907), 'Sur quelques propriétés des formes quadratiques positives parfaites', J. reine angew. Math. 133, 97-178.

Vice-Chancellor's Office

University of Adelaide

South Australia 